\title{
Phenotypical Effect of Phosphodiesterase 5 (PDE5) Inhibitor on Behavioral Activities of Fruit Fly Drosophila
} melanogaster

\author{
Azan Jaya ${ }^{1(\mathbb{D})}$, Elly Wahyudin 1(D), Yulia Yusrini Djabir ${ }^{1}{ }^{(\mathbb{D}}$, Tri Puspita Roska ${ }^{1}{ }^{(\mathbb{D}}$, \\ Rudi Arfiansyah ${ }^{1(\mathbb{D})}$, Andi Dirpan ${ }^{2}$, , Firzan Nainu 1,*(D) \\ 1 Faculty of Pharmacy, Universitas Hasanuddin, Makassar 90245, Indonesia \\ 2 Department of Agricultural Technology, Faculty of Agriculture, Universitas Hasanuddin, Makassar 90245, Indonesia \\ * Correspondence: firzannainu@unhas.ac.id;
}

Scopus Author ID 57120069200

Received: 28.02.2021; Revised: 30.03.2021; Accepted: 4.04.2021; Published: 19.04.2021

\begin{abstract}
Phosphodiesterase 5 (PDE5) inhibitor is a class of drugs currently used to treat erectile dysfunction. Physiologically, inhibition of PDE5 may lead to vasodilation, blood flow increment, and penile erection. However, PDE5 inhibitors have been reported not only to modify the function of the male reproductive organ but also to influence other physiological systems. To explore the effect of PDE5 inhibitor on metazoan physiological systems, a fruit fly (Drosophila melanogaster) model organism is used since the catalytic domain of fruit fly PDE5/6 shares a high similarity of amino acid sequence (58\%) with the PDE5 of humans. This study aimed to investigate whether the effect of PDE5 inhibition by sildenafil is phenotypically observable as changes in the behavioral states. Two behavioral phenotypes of D. melanogaster, negative geotaxis, and ethanol sensitivity, were used as test parameters in this explorative study. The results demonstrated that sildenafil had a significant effect on reducing locomotor activity, as reflected by negative geotaxis assay, but it had no influence on the fruit fly sensitivity to ethanol. Taken together, our results suggested that PDE5 inhibition might impair the physiological condition of the metazoan species. Also, an explorative study using D. melanogaster might offer valuable insight as a model organism in the discovery and repurposing approach of PDE5 inhibitor.
\end{abstract}

Keywords: phosphodiesterase 5; sildenafil; phenotypical; fruit fly; behavior activities.

(C) 2021 by the authors. This article is an open-access article distributed under the terms and conditions of the Creative Commons Attribution (CC BY) license (https://creativecommons.org/licenses/by/4.0/).

\section{Introduction}

Phosphodiesterase (PDE) is an enzyme that regulates cAMP and/or cGMP degradation at the cellular level [1]. Of the 11 classes of PDE found in humans, some are targeted for drugs, including phosphodiesterase 5 (PDE5) [2,3]. One of the pharmacological applications related to PDE5 is the use of PDE5 inhibitors to treat erectile dysfunction (ED) [4, 5]. Since PDE5 inhibitors have a similar structure with cGMP, these drugs competitively bind to PDE5 with cGMP, thus preventing cGMP degradation process [3, 6], which phenotypically observable as the state of penile erection [4]. At present, the most commonly used PDE5 inhibitor is sildenafil [4]. FDA approved this drug in 1998 following the success of clinical trials to demonstrate the efficacy of sildenafil in the treatment of ED [5,6]. Also, sildenafil has been used to treat pulmonary hypertension due to its pharmacological properties to increase nitric oxide concentration, leading to vasodilation in the lung [7-9]. 
At present, several PDE5 inhibitors, for example, sildenafil, tadalafil, and vardenafil have been widely marketed to treat ED [4, 10]. Apart from such main indication, PDE5 inhibitors were previously reported to yield anti-inflammatory [11] and antioxidant $[12,13]$ properties, further suggesting the possibility to repurpose PDE5 inhibitors into drugs to treat different diseases. Recently, PDE5 inhibitors have been suggested to be used in the treatment of Alzheimer's disease [14] and potentially beneficial in the management of Coronavirus disease (COVID)-19 [15]. However, much has not been known regarding the overall effect of PDE5 inhibitors on the human body, likely due to the difficulties in performing experimental research using human subjects. Also, our current pre-clinical platform using different types of mammalian animal models or even non-human primates' present significant challenges to drug repurposing efforts, possibly due to the necessity of ethical clearance, the complexity of signaling pathways involved in such models, or at the very least, economic problems [16]. To overcome those hurdles, developing an in vivo platform system that is easier to use, faster, and economical is urgently required [16]. With a high degree of genetic similarity to humans (genetically-homolog), the fruit fly Drosophila melanogaster is highly likely to serve as a prospective model organism in this research field.

The fruit fly, Drosophila melanogaster, is one of the model organisms that has been commonly used to explore the nature of numerous biological and physiological events [16-18], ranging from the investigation on the etiology and mechanistic basis of human diseases [19] to elucidation of the possible roles of an immune system malfunction in the emergence of sterile inflammation [20, 21]. Furthermore, due to the high similarity of genetic makeup (about 75\%) between $D$. melanogaster and human [16], this insect has been used in the pharmacological testing of drug candidates from natural products [22-25]. Additional advantages of using $D$. melanogaster as an in vivo model system are a very fast life cycle (around 2 months) with relatively lower maintenance costs, the availability of diverse types of mutant flies, and the possibility of using large numbers of flies at once with less ethical issues [16, 18]. A particular point of view that agrees with our research's purpose is $D$. melanogaster has been reported to have PDE5/6 that is homologous to the PDE5 of humans [26].

Drosophila melanogaster has five PDE types, namely PDE1, PDE5/6, PDE8, PDE9, and PDE11 [26]. Sildenafil, a widely used PDE5 inhibitor, has interacted with the PDE 5/6 of D. melanogaster [26]. However, the effects of such drug-enzyme interaction on behavioral phenotypes of $D$. melanogaster have not been reported yet. To explore the effect of PDE5 inhibitors on metazoan behaviors, we used fruit fly $D$. melanogaster as the model organism since the catalytic domain of fruit fly PDE5/6 shared a high similarity of amino acid sequence (58\%) with the PDE5 and the PDE6 (51\%) of humans [26]. In this study, two behavioral properties of $D$. melanogaster, negative geotaxis and sensitivity of fly to ethanol, were used to observe the effect of sildenafil on Drosophila behaviors. Negative geotaxis is a term used to reflect the upward vertical movement of fruit fly in the vial [27], and the ethanol sensitivity assay is widely used to assess the effect of certain stimulation/destimulation on the central nervous system of Drosophila concerning its locomotor [28]. The purpose of these tests was to determine whether Drosophila can experience changes in either locomotor speed or ethanol sensitivity after the drug has been introduced. This study serves as a proof-of-concept proxy to investigate behavioral changes of $D$. melanogaster in response to the introduction of sildenafil, a widely used PDE5 inhibitor. 


\section{Materials and Methods}

\subsection{Fly stock maintenance and treatment.}

Males of the Drosophila melanogaster $w^{1118}$ line were used in the entire experiment performed in this study. Males of $D$. melanogaster were discriminated from their females' counterparts by straightforward observation on the genital and dorsal-posterior features (segments A5 and A6) of the corresponding flies using a stereomicroscope.

Fruit fly D. melanogaster was divided into five groups. Each group consisted of ten male flies at the age of 1-2 days determined from their pupal case's first day of emergence. Flies in the healthy control group were fed with standard food, and flies in the vehicle control group were incubated in a mixture of standard food and DMSO. Treatment groups were fed with standard food containing sildenafil at concentrations of either $1 \mu \mathrm{M}, 10 \mu \mathrm{M}$, or $100 \mu \mathrm{M}$. Each group was treated for five days, and flies were maintained in standard conditions $\left(25^{\circ} \mathrm{C}\right.$, 12 hours light, and 12 hours dark cycles) until the end of observation.

\subsection{Locomotor assay.}

All fly groups were incubated at $25^{\circ} \mathrm{C}$ and monitored for locomotor (Fig. 1). Locomotor testing was performed by a negative geotaxis method on flies placed in a marked empty vial, designated as the locomotor testing vial, as described previously [29], with slight modifications. The testing vial was placed in an upright standing position in front of a climbing wall. Briefly, this test was started by firmly tapping off the locomotor testing vial at the start to ensure all flies started the movement from the bottom of the vial. To obtain the data, we observed and recorded events that happened in the testing vial for up to 30 seconds. Flies were assigned based on their position on the climbing wall, and the data were further analyzed using a custom-made Excel spreadsheet as used in [29].

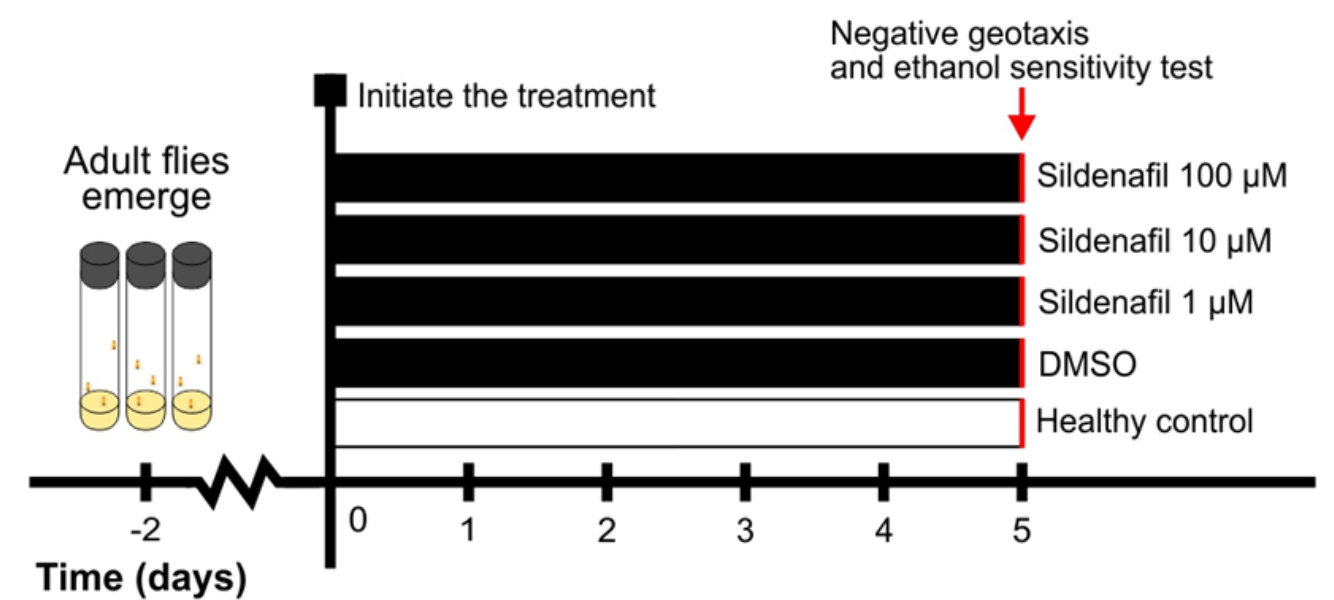

Figure 1. Timeline of the experimental design. Adult flies of each group were given normal fly food or fly food containing either sildenafil with different concentrations of DMSO. Fly locomotor was assessed using negative geotaxis assay after five days of treatment in the presence or absence of sildenafil at different concentrations. The end of each bar means all flies in that group were subjected to negative geotaxis and ethanol sensitivity assay. DMSO, dimethyl sulfoxide

\subsection{Ethanol sensitivity assay.}

All fly groups were incubated at $25^{\circ} \mathrm{C}$ and monitored for their ethanol sensitivity (Fig. 1). Ethanol sensitivity assay was conducted on flies placed in marked empty vials (in the absence of fly food), as described previously [30], with slight modifications. Briefly, ten males 
of D. melanogaster at the age of 1-2 days were used in this assay. These flies were placed in an empty vial, designated as the ethanol testing chamber, for several minutes for adaptation and then subjected to the ethanol exposure protocol. At the end of the experiment, flies that were still alive after being exposed to the $96 \%$ ethanol were then counted. Results obtained from each group were then analyzed and compared statistically.

\subsection{Data analysis.}

All data obtained in this study were subjected to statistical analysis using the GraphPad Prism ${ }^{\circledR} 8$ application, based on a One-Way ANOVA followed by a suitable post hoc approach. For all statistical analyses, data were presented as mean \pm S.D., and $p$ values of less than 0.05 were considered statistically significant.

\section{Results and Discussion}

\subsection{Effect of sildenafil on the locomotor of Drosophila.}

Locomotor testing was performed to observe changes in the Drosophila movement after sildenafil ingestion at different concentrations. In humans, sildenafil has been shown to increase protein synthesis in the muscle, thus presumably leads to the reduction of muscle fatigue [31]. Therefore, we hypothesized that Drosophila locomotor might be increased or steadily maintained in response to sildenafil ingestion in Drosophila. To test this hypothesis, we carried out a negative geotaxis assay. Results obtained from this experiment (Fig. 2) showed that the locomotor of flies in the groups of sildenafil-treated Drosophila at concentrations of $10 \mu \mathrm{M}$ and $100 \mu \mathrm{M}$ was reduced compared to the healthy control or the DMSO control, suggesting that Drosophila locomotor was negatively impaired in the presence of sildenafil. Interestingly, this result was different from our expectation, suggesting that our findings in Drosophila might not result in an agreement to the results previously obtained from humans [31]. Mechanisms on how sildenafil can impair fly locomotor in such trend, as measured using negative geotaxis assay, remain elusive. Possible explanations for such events might be related to the local influence of sildenafil on Drosophila muscle cells or a more systematic effect of the drug on the fly central nervous system (CNS). Either way or another, this might lead to impairment in the fly locomotor ability. However, this remains speculative as more experiments are required to answer this question.

A

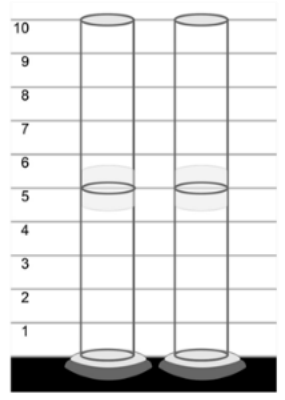

B

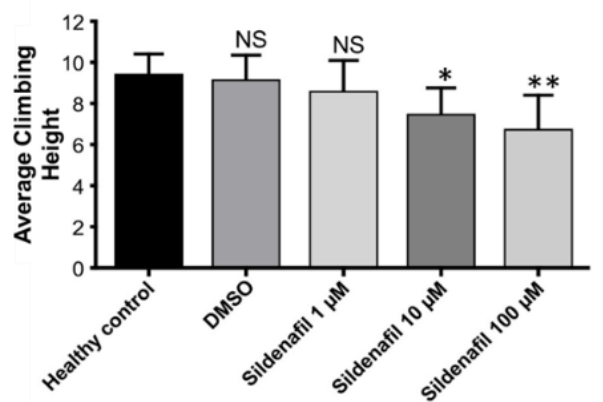

Figure 2. Negative geotaxis assay on the males of $w^{1118}$ Drosophila in the presence or absence of sildenafil. (A) Design of fly climbing wall and testing vials used in the negative geotaxis assay. (B) Results obtained from negative geotaxis assay. Adult flies at the age of 1-2 days were fed with fly food containing either $1 \mu \mathrm{M}, 10 \mu \mathrm{M}$, or $100 \mu \mathrm{M}$ sildenafil. Flies incubated in DMSO-containing fly food were used as the vehicle control in this experiment. All flies were subjected to a negative geotaxis assay, and results were analyzed accordingly. DMSO, dimethyl sulfoxide; Statistical significance $*=p<0.05, * *=p<0.01, \mathrm{NS}=$ not significant. 


\subsection{Effect of sildenafil on the sensitivity of Drosophila to ethanol.}

Drosophila has been known to be attracted to foods containing a low level of alcohol, mainly in the form of ethanol, as a result of the fermentation process [32, 33]. Ethanol itself has been reported to induce genetic and phenotypical changes in Drosophila [34]. A high level of ethanol could promote the death of exposed Drosophila [35], similar to those reported in humans [36]. In light of results obtained from previous experiments, D. melanogaster has been suggested as a suitable model organism to study the genetics of alcoholism in humans [37, 38].

A previous experiment on eight healthy male subjects demonstrated that sildenafil and alcohol (in the form of red wine) did not show important hemodynamic interaction clinically [39], suggesting the safe use of sildenafil under alcohol presence. Alternatively, one may assume that sildenafil and alcohol might involve a complex interaction in the metazoan biochemical process. Previous studies have shown that sildenafil could induce the expression of endogenous antioxidants, such as superoxide dismutases and catalases, in healthy men [40] and rodent [41] subjects. While the detail of the mechanisms remains elusive, it is tempting to speculate that increased expression of endogenous antioxidants can prevent negative alcohol effects on the metazoan cells.

Ethanol sensitivity assay has been demonstrated as one of the easiest and cheapest methods to examine certain exogenous substances' effect on fly behavior [28, 30, 42]. Owing to that, we intended to assess whether oral administration of sildenafil into flies would induce changes in flies' behavior towards ethanol. To achieve that, we performed an ethanol sensitivity assay using methods described previously [30]. As seen in Fig. 3, the results showed that oral administration of sildenafil at different concentrations before ethanol sensitivity assay did not change the time flies succumbed to ethanol, suggesting that sildenafil treatment did not induce phenotypical changes in Drosophila sensitivity to ethanol. In the healthy control group, the time required for Drosophila to undergo $100 \%$ ethanol-induced fly death was about 733 seconds, while flies treated with sildenafil per oral at concentrations of $1 \mu \mathrm{M}, 10 \mu \mathrm{M}$, or 100 $\mu \mathrm{M}$, underwent $100 \%$ death at around 700 seconds. Moreover, all flies in the negative control group also similarly underwent mortality at around 650 seconds. Statistically, there is no significant difference in terms of fly behavior towards ethanol from one group to others, suggesting that administration of sildenafil to flies has less or even no effect on the fly sensitivity to ethanol.

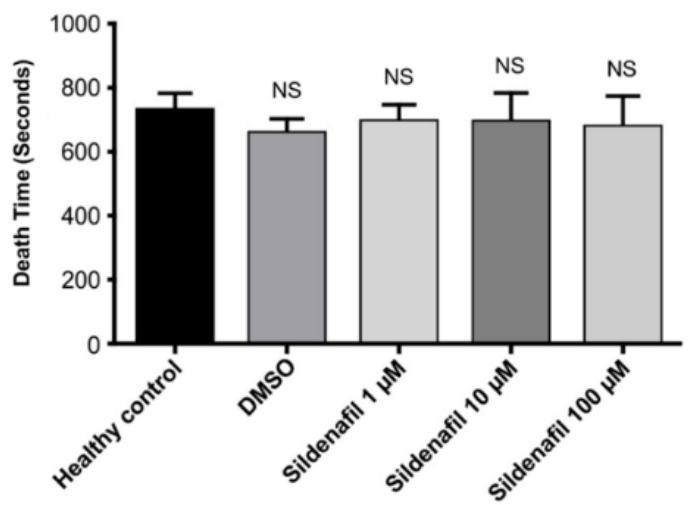

Figure 3. Result of ethanol sensitivity assay on the males of $w^{1118}$ Drosophila in the presence or absence of sildenafil. Adult flies at the age of 1-2 days were fed with fly food containing either $1 \mu \mathrm{M}, 10 \mu \mathrm{M}$, or $100 \mu \mathrm{M}$ sildenafil. Flies incubated in DMSO-containing fly food were used as the vehicle control in this experiment. All flies were subjected to ethanol sensitivity assay, and results were analyzed accordingly. DMSO, dimethyl sulfoxide; Statistical significance $*=p<0.05, * *=p<0.01$, NS $=$ not significant. 
It is important to note that the test was performed by pipetting the ethanol on the vial cap as described previously [30]; thus, ethanol might fill in space in the vial, thus reducing the chance of ethanol to yield the expected effect to Drosophila. Nevertheless, our results in this study provide a hint for sildenafil effect on Drosophila behaviors previously unaware. We believe that the number of phenotypical characteristics to be observed in the future study shall be increased with this preliminary data. It might be possible to use phenotypical characteristics as high-throughput screening parameters in the discovery of new PDE5 inhibitors or drug repurposing campaigns in the long run.

\section{Conclusions}

Based on our results, we concluded that the oral administration of sildenafil, a PDE 5 inhibitor, at a concentration of $100 \mu \mathrm{M}$ resulted in reducing Drosophila locomotor but had no effect on the Drosophila sensitivity to ethanol. In the long run, these preliminary data, in addition to results obtained from other behavioral assays, might hold a potential impact on the PDE5 inhibitors repurposing effort.

\section{Funding}

This research received Hasanuddin University funding (Hibah Penelitian Skim Professorship to E.W., F.N., and Y.Y.D; No. 2419/UN4.21.1/TU.21/2017).

\section{Acknowledgments}

We want to offer our appreciation to Prof. Yoshinobu Nakanishi (Kanazawa University, Japan) for his kind support in providing the Drosophila line used in this study.

\section{Conflicts of Interest}

The authors declare no conflict of interest.

\section{References}

1. Bender, A.T.; Beavo, J.A. Cyclic Nucleotide Phosphodiesterases: Molecular Regulation to Clinical Use. Pharmacol Rev 2006, 58, 488-520, https://doi.org/10.1124/pr.58.3.5.

2. Omori, K.; Kotera, J. Overview of PDEs and their regulation. Circ Res 2007, 100, 309-327, https://doi.org/10.1161/01.RES.0000256354.95791.f1.

3. Ahmed, W.S.; Geethakumari, A.M.; Biswas, K.H. Phosphodiesterase 5 (PDE5): Structure-function regulation and therapeutic applications of inhibitors. Biomedicine \& Pharmacotherapy 2021, 134, https://doi.org/10.1016/j.biopha.2020.111128.

4. Huang, S.A.; Lie, J.D. Phosphodiesterase-5 (PDE5) Inhibitors In the Management of Erectile Dysfunction. $P$ T 2013, 38, 407-419.

5. $\quad$ Dong, L.; Zhang, X.; Yan, X.; Shen, Y.; Yu, X.; Li, Y. Effect of phosphodiesterase-5 inhibitors (PDE5is) on the treatment of male infertility: A protocol for systematic review and meta-analysis. Medicine (Baltimore) 2019, 98, https://doi.org/10.1097/md.0000000000018317.

6. Boswell-Smith, V.; Spina, D.; Page, C.P. Phosphodiesterase inhibitors. British journal of pharmacology 2006, 147 Suppl 1, S252-S257, https://doi.org/10.1038/sj.bjp.0706495.

7. Galiè, N.; Ghofrani, H.A.; Torbicki, A.; Barst, R.J.; Rubin, L.J.; Badesch, D.; Fleming, T.; Parpia, T.; Burgess, G.; Branzi, A.; Grimminger, F.; Kurzyna, M.; Simonneau, G. Sildenafil citrate therapy for pulmonary arterial hypertension. $N$ Engl J Med 2005, 353, 2148-2157, https://doi.org/10.1056/NEJMoa050010.

8. Andersson, K.E. PDE5 inhibitors - pharmacology and clinical applications 20 years after sildenafil discovery. British journal of pharmacology 2018, 175, 2554-2565, https://doi.org/10.1111/bph.14205.

9. Pich, J. Phosphodiesterase 5 inhibitors for pulmonary hypertension. Int J Evid Based Healthc 2020, 18, 155156, https://doi.org/10.1097/xeb.0000000000000198. 
10. Madeira, C.R.; Tonin, F.S.; Fachi, M.M.; Borba, H.H.; Ferreira, V.L.; Leonart, L.P.; Bonetti, A.F.; Moritz, R.P.; Trindade, A.; Gonçalves, A.G.; Fernandez-Llimos, F.; Pontarolo, R. Efficacy and safety of oral phosphodiesterase 5 inhibitors for erectile dysfunction: a network meta-analysis and multicriteria decision analysis. World J Urol 2020, 39, 953-962, https://doi.org/10.1007/s00345-020-03233-9.

11. Peixoto, C.A.; Gomes, F.O.D.S. The role of phosphodiesterase-5 inhibitors in prostatic inflammation: a review. J Inflamm (Lond) 2015, 12, 54-54, https://doi.org/10.1186/s12950-015-0099-7.

12. Tanus-Santos, J.E. Antioxidant effects of phosphodiesterase-5 inhibitors. Cardiovascular Research 2013, 100, 170-170, https://doi.org/10.1093/cvr/cvt189.

13. Fan, Y.F.; Zhang, R.; Jiang, X.; Wen, L.; Wu, D.C.; Liu, D.; Yuan, P.; Wang, Y.L.; Jing, Z.C. The phosphodiesterase-5 inhibitor vardenafil reduces oxidative stress while reversing pulmonary arterial hypertension. Cardiovasc Res 2013, 99, 395-403, https://doi.org/10.1093/cvr/cvt109.

14. Zuccarello, E.; Acquarone, E.; Calcagno, E.; Argyrousi, E.K.; Deng, S.X.; Landry, D.W.; Arancio, O.; Fiorito, J. Development of novel phosphodiesterase 5 inhibitors for the therapy of Alzheimer's disease. Biochem Pharmacol 2020, 176, https://doi.org/10.1016/j.bcp.2020.113818.

15. Giorgi, M.; Cardarelli, S.; Ragusa, F.; Saliola, M.; Biagioni, S.; Poiana, G.; Naro, F.; Massimi, M. Phosphodiesterase Inhibitors: Could They Be Beneficial for the Treatment of COVID-19? International Journal of Molecular Sciences 2020, 21, https://doi.org/10.3390/ijms21155338.

16. Pandey, U.B.; Nichols, C.D. Human disease models in Drosophila melanogaster and the role of the fly in therapeutic drug discovery. Pharmacol Rev 2011, 63, 411-436, https://doi.org/10.1124/pr.110.003293.

17. Ugur, B.; Chen, K.; Bellen, H.J. Drosophila tools and assays for the study of human diseases. Dis Model Mech 2016, 9, 235-244, https://doi.org/10.1242/dmm.023762.

18. Nainu, F.; Nakanishi, Y.; Shiratsuchi, A. Fruit fly as a model organism in the study of human diseases and drug discovery. Journal of Center for Medical Education Sapporo Medical University 2019, 21-32.

19. Chow, C.Y.; Reiter, L.T. Etiology of Human Genetic Disease on the Fly. Trends Genet 2017, 33, 391-398, https://doi.org/10.1016/j.tig.2017.03.007.

20. Nainu, F.; Salim, E.; Asri, R. M.; Hori, A.; Kuraishi, T. Neurodegenerative disorders and sterile inflammation: lessons from a Drosophila model. The Journal of Biochemistry 2019, 166, 213-221, https://doi.org/10.1093/jb/mvz053.

21. Asri, R.M.; Salim, E.; Nainu, F.; Hori, A.; Kuraishi, T. Sterile induction of innate immunity in Drosophila melanogaster. Frontiers in Bioscience (Landmark Edition) 2019, 24, 1390-1400,

22. Ahsan, M.; Gonsales, A.V.; Sartini, S.; Wahyudin, E.; Nainu, F. In vivo anti-staphylococcal activity of roselle (Hibiscus sabdariffa L.) calyx extract in Drosophila model of infection. Journal of Herbmed Pharmacology 2019, 8, 41-46, https://doi.org/10.5530/pj.2018.5.169.

23. Nainu, F.; Asri, R.M.; Arsyad, A.; Manggau, M.A.; Amir, M.N. In vivo antibacterial activity of green algae Ulva reticulata against Staphylococcus aureus in Drosophila model of infection. Pharmacognosy Journal 2018, 10, 993-997, https://doi.org/10.5530/pj.2018.5.169.

24. Nainu, F.; Asri, R.M.; Djide, M.N.; Ahsan, M.; Arfiansyah, R.; Sartini, S.; Alam, G. Protective effect of green algae Ulva reticulata against Pseudomonas aeruginosa in Drosophila infection model. HAYATI Journal of Biosciences 2019, 26, 163-171.

25. Nainu, F.; Djide, M. N.; Subehan, S.; Sartini, S.; Roska, T. P.; Salim, E.; Kuraishi, T. Protective Signatures of Roselle (Hibiscus sabdariffa L.) Calyx Fractions against Staphylococcus aureus in Drosophila Infection Model. HAYATI Journal of Biosciences 2020, 27, 306-313.

26. Day, J.P.; Dow, J.A.; Houslay, M.D.; Davies, S.A. Cyclic nucleotide phosphodiesterases in Drosophila melanogaster. Biochem J 2005, 388, 333-342, https://doi.org/10.1042/bj20050057.

27. Linderman, J.A.; Chambers, M.C.; Gupta, A.S.; Schneider, D.S. Infection-related declines in chill coma recovery and negative geotaxis in Drosophila melanogaster. PLoS One 2012, 7, https://doi.org/10.1371/journal.pone.0041907.

28. Rothenfluh, A.; Heberlein, U. Drugs, flies, and videotape: the effects of ethanol and cocaine on Drosophila locomotion. Curr Opin Neurobiol 2002, 12, 639-645, https://doi.org/10.1016/s0959-4388(02)00380-x.

29. Strangward, P.; Alnuamaani, C.; Patel, S.; Prokop, A. Lesson 1 - The climbing assay: Learning data analysis through live experiments with fruit flies. In: Droso4Schools.wordpress.com, (Manchester, U.K: Machester Fly Facility). Accessed on $20^{\text {th }}$ July 2020.

30. Maples, T.; Rothenfluh, A. A simple way to measure ethanol sensitivity in flies. J Vis Exp 2011, 2541, https://doi.org/10.3791/2541.

31. Sheffield-Moore, M.; Wiktorowicz, J.E.; Soman, K.V.; Danesi, C.P.; Kinsky, M.P.; Dillon, E.L.; Randolph, K.M.; Casperson, S.L.; Gore, D.C.; Horstman, A.M.; Lynch, J.P.; Doucet, B.M.; Mettler, J.A.; Ryder, J.W.; Ploutz-Snyder, L.L.; Hsu, J.W.; Jahoor, F.; Jennings, K.; White, G.R.; McCammon, S.D.; Durham, W.J. Sildenafil increases muscle protein synthesis and reduces muscle fatigue. Clin Transl Sci 2013, 6, 463-468, https://doi.org/10.1111/cts.12121.

32. Pohl, J.B.; Baldwin, B.A.; Dinh, B.L.; Rahman, P.; Smerek, D.; Prado, F.J., 3rd; Sherazee, N.; Atkinson, N.S. Ethanol preference in Drosophila melanogaster is driven by its caloric value. Alcohol Clin Exp Res 2012, 36, 1903-1912, https://doi.org/10.1111/j.1530-0277.2012.01817.x.

33. Markow, T.A. The secret lives of Drosophila flies. eLife 2015, 4, https://doi.org/10.7554/eLife.06793. 
34. Park, A.; Ghezzi, A.; Wijesekera, T.P.; Atkinson, N.S. Genetics and genomics of alcohol responses in Drosophila. Neuropharmacology 2017, 122, 22-35, https://doi.org/10.1016/j.neuropharm.2017.01.032.

35. Scholz, H.; Ramond, J.; Singh, C.M.; Heberlein, U. Functional Ethanol Tolerance in Drosophila. Neuron 2000, 28, 261-271, https://doi.org/10.1016/S0896-6273(00)00101-X.

36. Witkiewitz, K.; Litten, R.Z.; Leggio, L. Advances in the science and treatment of alcohol use disorder. Sci Adv 2019, 5, https://doi.org/10.1126/sciadv.aax4043.

37. Lathen, D.R.; Merrill, C.B.; Rothenfluh, A. Flying Together: Drosophila as a Tool to Understand the Genetics of Human Alcoholism. Int J Mol Sci 2020, 21, https://doi.org/10.3390/ijms21186649.

38. Engel, G.L.; Taber, K.; Vinton, E.; Crocker, A.J. Studying alcohol use disorder using Drosophila melanogaster in the era of 'Big Data'. Behav Brain Funct 2019, 15, https://doi.org/10.1186/s12993-0190159-x.

39. Leslie, S.J.; Atkins, G.; Oliver, J.J.; Webb, D.J. No adverse hemodynamic interaction between sildenafil and red wine. Clin Pharmacol Ther 2004, 76, 365-370, https://doi.org/10.1016/j.clpt.2004.07.005.

40. Perk, H.; Armagan, A.; Naziroğlu, M.; Soyupek, S.; Hoscan, M.B.; Sütcü, R.; Ozorak, A.; Delibas, N. Sildenafil citrate as a phosphodiesterase inhibitor has an antioxidant effect in the blood of men. J Clin Pharm Ther 2008, 33, 635-640, https://doi.org/10.1111/j.1365-2710.2008.00962.x.

41. Sheweita, S.; Salama, B.; Hassan, M. Erectile dysfunction drugs and oxidative stress in the liver of male rats. Toxicol Rep 2015, 2, 933-938, https://doi.org/10.1016/j.toxrep.2015.06.002.

42. Rodan, A.R.; Rothenfluh, A. The genetics of behavioral alcohol responses in Drosophila. Int Rev Neurobiol 2010, 91, 25-51, https://doi.org/10.1016/S0074-7742(10)91002-7. 